\title{
Sensitivity Analysis of a Fully Kinetic Code for Physical Parameters*
}

\author{
Burak KARADAG, ${ }^{1 \dagger \dagger}$ Shinatora CHO, ${ }^{2)}$ and Ikkoh FUNAKI ${ }^{1), 2)}$ \\ ${ }^{1)}$ The Graduate University for Advanced Studies (SOKENDAI), Sagamihara, Kanagawa 252-5210, Japan \\ ${ }^{2)}$ Japan Aerospace Exploration Agency (JAXA), Sagamihara, Kanagawa 252-5210, Japan
}

\begin{abstract}
A sensitivity analysis of a fully kinetic particle code was conducted to investigate the importance of uncertainties associated to physical parameters. A $500 \mathrm{~W}$-class laboratory model magnetic-layer Hall thruster was used as the testbed. The sensitivities of the physical parameters, including thermal accommodation coefficient, anode/wall temperature, Bohm diffusion coefficient, electron injection current, cathode coupling voltage, and background pressure, were quantified one-byone on a conservative possible range. The results suggest the wall erosion prediction is more sensitive to the physical parameters than the thrust or the discharge current. Among the physical parameters, sensitivity to the Bohm diffusion coefficient and parameters related to the neutral flow (i.e., thermal accommodation coefficient and anode/wall temperatures) were dominant. It was hence found that uncertainties in the physical parameters related to the neutral flow had comparable influence on the Bohm diffusion coefficient despite the low attention they attracted.
\end{abstract}

Key Words: Kinetic Simulation, Sensitivity Analysis, Hall Thruster

\section{Nomenclature}

$B$ : magnetic field strength

$B_{r}$ : radial magnetic field strength

$D_{\perp}:$ cross-field diffusion coefficient

$e$ : unit charge

$F$ : thrust

$I_{b}$ : beam current

$I_{b 1}$ : singly charged ion current

$I_{b 2}$ : doubly charged ion current

$I_{d}$ : discharge current

$I_{g}$ : magnetic pole current

$I_{n}$ : unionized neutral propellant current

$k_{B}$ : Boltzmann constant

$m$ : electron mass

$\dot{m}$ : propellant mass flow rate

$P_{j e t}$ : energy deposited on propellant

$P_{d}$ : discharge power

$T_{\text {anode }}$ : anode temperature

$T_{\text {wall }}$ : inner and outer wall temperature

$T_{e}$ : electron temperature

$V_{b}$ : beam voltage

$V_{c}$ : cathode coupling voltage

$V_{d}$ : discharge voltage

$v$ : speed

$\Delta$ : discharge current oscillation amplitude

$\eta_{t}:$ anode/thrust efficiency

$\eta_{E}$ : energy efficiency

$v$ : electron collision frequency

$\eta_{u}$ : propellant utilization efficiency

(C) 2017 The Japan Society for Aeronautical and Space Sciences *Preliminary results were presented at Joint Conference of 30th ISTS/ 34th IEPC \& 6th NSAT, 4-10 July 2015, Kobe, Japan

Received 16 November 2015; final revision received 30 September 2016; accepted for publication 25 October 2016.

${ }^{\dagger}$ Corresponding author, burak.karadag@ac.jaxa.jp $\eta_{a}$ : beam efficiency

$\omega_{c}$ : electron cyclotron frequency

\section{Introduction}

A Hall thruster ${ }^{1}$ is a type of electric propulsion used for satellite missions like station-keeping, orbit transfer or deep space exploration. Hall thrusters are preferable due to many advantages, such as high thrust density (no space-charge limitation defined by Child's law as ions are accelerated in quasi-neutral plasma), moderate specific impulse, and outstanding in-space experience. To improve/optimize the performance of Hall thrusters and understand discharge chamber plasma physics, numerical simulations are important in that they are cheaper and faster compared to experiments and they provide physical information often difficult to obtain from experiments. For example, quantifying plasma properties inside the discharge chamber is a difficult task as the interaction of probes with highly energetic particles would affect the measurement result. For the present, numerical simulations are only assistive methods and unlikely to replace experiments since complex plasma behavior is far from being well understood.

There are three main approaches (fluid, fully kinetic, and hybrid) in modeling Hall thrusters. ${ }^{2-4)}$ Fluid codes treat both electrons and ions as fluid and assume Maxwellian velocity distribution function (VDF). ${ }^{5)}$ Fully kinetic codes treat electrons and ions as discrete particles and compute VDF selfconsistently. ${ }^{6}$ As for hybrid codes, electrons are treated as a fluid, whereas ions are treated as particles, compromising the difference between fluid and kinetic approaches. ${ }^{7,8)}$ Fully kinetic codes are computationally expensive and time-consuming; however, they can capture non-equilibrium features unlike the fluid and hybrid codes. For instance, plasma-wall sheath is self-consistently modeled in fully kinetic codes, while a sheath model is required for fluid and hybrid codes. 
It is therefore expected that fully kinetic simulation is potentially capable of high-accuracy predictive modeling. Nevertheless, prediction accuracy of fully kinetic codes is decreased by various sources of uncertainty and error, which involves both numerical discretization errors and uncertainties in model physical parameters. It is important to investigate these errors/uncertainties and quantify their confidence range for the realization of predictive modeling. However, there are few previous studies on this subject so far, especially for the physical parameter uncertainties of fully kinetic simulation.

This study serves as the first step of uncertainty quantification of fully kinetic Hall thruster modeling. As for the sensitivity analysis, a one-factor-at-a-time sensitivity analysis method was preferred due to its simplicity. The one-factorat-a-time method involves repeatedly varying one parameter value in a presumed confidence range at a time while keeping others fixed at their nominal value. Previously, Watanabe et al. ${ }^{9}$ adopted this approach to conduct a sensitivity analysis of the input parameters of a numerical tool to validate grid erosion of an ion thruster. In the following sections, the results of our sensitivity analysis are presented, and the significance of the physical parameters is discussed.

\section{Numerical Methodology}

\subsection{Hall thruster}

A laboratory model magnetic-layer Hall thruster of The University of Tokyo named UT-62, ${ }^{10)}$ and having an outerchannel diameter of $62 \mathrm{~mm}$ was chosen to be the test bed. The electrical diagram of the experimental setup is shown in Fig. 1(a).

\subsection{Code description}

A 2D3V fully kinetic Particle-in-Cell (PIC)/Direct Simulation Monte Carlo (DSMC) code was used in this study. To compensate for the lower electron mobility across the magnetic field due to the lack of azimuthal resolution, Bohm diffusion was implemented in terms of virtual scattering collision $^{11)}$ between neutrals and electrons as $v_{\text {Bohm }}=$ $1 / 16\left(e B / m_{e}\right)$. The total collision frequency is defined as:

$$
v_{\text {total }}=v_{\text {elastic }}+v_{\text {ionization }}+v_{\text {excitation }}+v_{\text {Bohm }}
$$

To speed-up the code and make the simulation feasible, so-called macroparticles and an artificial mass ratio model were used. The magnetic field induced by the plasma was considered to be negligible. The magnetic field was calculated using Finite Element Method Magnetics (FEMM).

A uniform rectangular grid in a cylindrical coordinate system $(r, z, \theta)$ was adopted as shown in Fig. 1(b), and the cell interval was arranged in the same order as the dielectric wall sheath. A fixed potential was applied to the anode and the plume boundary, and the space potential $\varphi$ was calculated according to the Poisson's equation. A semi-implicit solver was used because it stabilizes the simulation even at larger time steps than electron plasma frequency and larger grid spacing than Debye length, thus remarkably reducing computational cost. The grid spacing was decided according to

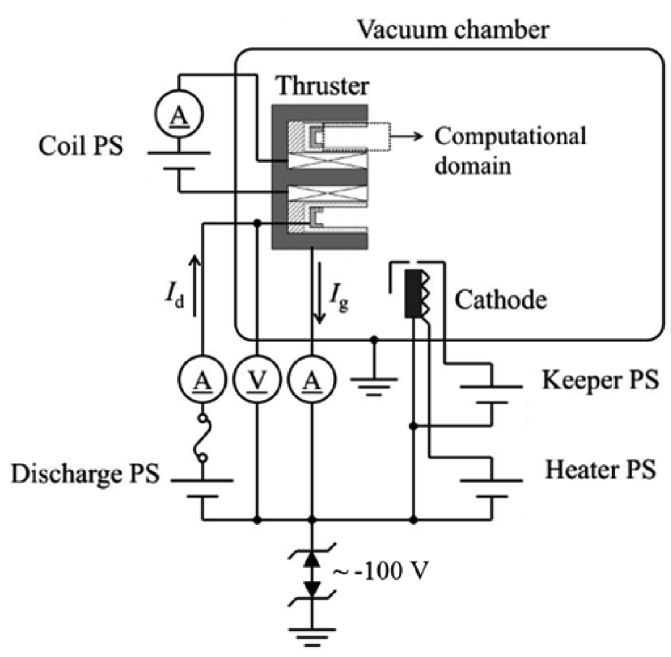

(a) Electrical diagram of the experimental setup. PS : power supply

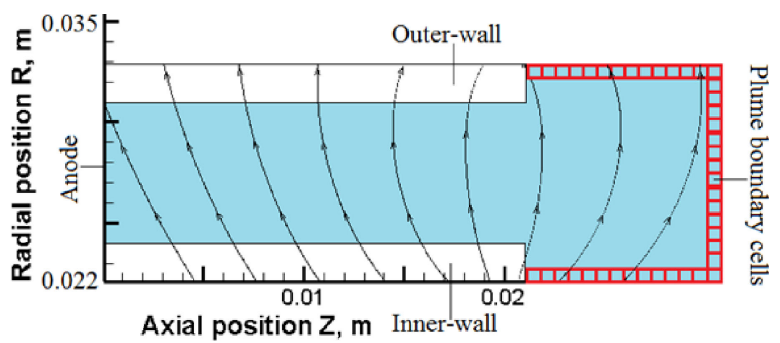

(b) Close-up view of computational domain. Lines with arrows represent the magnetic field applied

Fig. 1. UT-62 Hall thruster schematics.

the parametric study conducted by Cho et al. ${ }^{12)}$ Even though the Debye length $(\sim 0.05 \mathrm{~mm})$ is not resolved, plasma oscillations such as discharge current oscillation are captured by the semi-implicit solver. To calculate the particle trajectories of all species, the Lorentz force equation of motion is solved using the 4th-order Runge-Kutta method. Electric and magnetic forces were applied via the PIC method while inter-particle collisions were implemented using DSMC. Only singly charged ions, doubly charged ions, electrons, and neutrals were simulated. The computational time-step was chosen such that the plasma oscillation and electron cyclotron motion were captured.

The code was parallelized using the Message Passing Interface (MPI) and Portable, Extensible Toolkit for Scientific Computation (PETSc), and simulations were conducted using a quad-core $3.4 \mathrm{GHz} \mathrm{CPU}$ personal computer. Further details of the simulation methodology can be found elsewhere. ${ }^{12,13)}$

\subsection{Simulation conditions}

The discharge voltage, mass flow rate, and coil current were set to $300 \mathrm{~V}, 1 \mathrm{~A}_{\mathrm{eq}}(1.361 \mathrm{mg} / \mathrm{s}$ for Xenon), and $2 \mathrm{~A}$ respectively. Figure 2 plots the radial magnetic field strength distribution along the inner/outer wall surfaces and discharge channel centerline for a $2 \mathrm{~A}$ coil current. Magnetic field strength varied between $0.005-0.03$ Tesla along the discharge channel centerline and its strength increased from the anode towards the thruster exit. Simulations for each case 


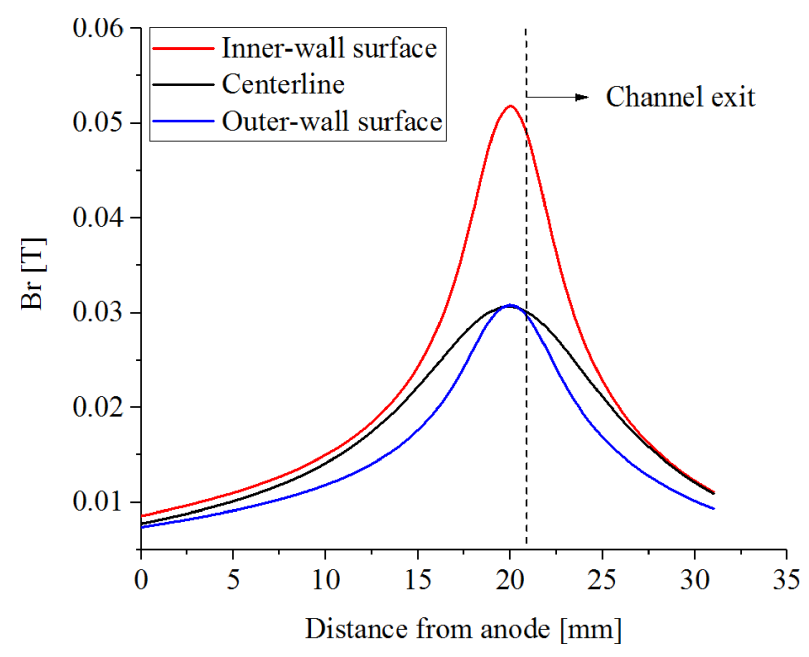

Fig. 2. Radial magnetic field distribution.

were run for 0.4 million steps. All of the performance results and plasma distribution plots were time-averaged. Table 1 lists simulation nominal physical parameters. Additionally, the measurement results of discharge current, thrust, and anode efficiency were tabulated. ${ }^{12)}$

All of the simulation results were time-averaged over $0.1 \mathrm{~ms}$ samplings every 100 simulation steps. The convergence of the simulation was judged by the time-history of the total number of simulation particles. The efficiency analysis scheme of Brown ${ }^{14)}$ was used to investigate the details of thruster performance (see Appendix).

\subsection{Physical parameters investigated in this study}

The physical parameters investigated in this study involve the thermal accommodation coefficient of heavy particles, anode temperature, wall temperature, Bohm diffusion coefficient, cathode coupling potential drop, electron injection current to the computational domain, and background pressure effect. This set of parameters essentially comprises all of the physical parameters of our fully kinetic model, and each of them can have a non-trivial influence on the simulation results. The thermal accommodation coefficient and anode/ wall temperatures are expected to change the neutral flow inside the discharge channel. ${ }^{15)}$ The noble Bohm diffusion coefficient is known to dominate the electron cross-field transport. ${ }^{16)}$ Cathode potential drop and electron injection current are related to the thruster-cathode coupling, which can mainly influence the voltage utilization of ions. ${ }^{17)}$ Additionally, the background pressure effect ${ }^{18)}$ is attracting increasing attention from the Hall thruster community ${ }^{19)}$ though it is an uncertainty of the experiment rather than simulation. Uncertainties in all of these physical parameters can be categorized under epistemic uncertainty ${ }^{20}$ or systematic error, which means there is a true deterministic value for each parameter, although we do not know about its exact value due to lack of accessibility (i.e., unable to measure, measurement error, etc.).

In numerical simulation, the deficiencies of the models can be divided into two subcategories: 1) Errors associated with numerical methods, and 2) uncertainties in code input param-
Table 1. Simulation conditions.

\begin{tabular}{lll}
\hline Thruster operation conditions & Discharge voltage [V] & 300 \\
& Xe mass flow rate $[\mathrm{mg} / \mathrm{s}]$ & 1.36 \\
& Magnetic coil current $[\mathrm{A}]$ & 2.0 \\
\hline Numerical settings & Time step [s] & $5 \times 10^{-10}$ \\
& Macroparticle size & $1 \times 10^{7}$ \\
& Mass ratio $m_{i} / m_{e}$ & 96.4 \\
& Grid spacing [mm] & 0.2 \\
\hline Experimental results & Discharge current $[\mathrm{A}]$ & 0.99 \\
& Thrust [mN] & 16.3 \\
& Anode efficiency [\%] & 32.6 \\
\hline
\end{tabular}

Table 2. Physical parameters investigated. A: Thermal accommodation coefficient and anode/wall temperatures, B: Bohm diffusion coefficient, C: Cathode coupling voltage, E: Electron injection current, and BP: Background pressure effect.

\begin{tabular}{llccc}
\hline \multicolumn{2}{c}{ Parameter } & Minimum & Nominal & Maximum \\
\hline A & TAC & 0.5 & 0.9 & 1.0 \\
& $T_{\text {anode }}[\mathrm{K}]$ & 900 & 500 & 500 \\
& $T_{\text {wall }}[\mathrm{K}]$ & 900 & 700 & 500 \\
$\mathrm{~B}$ & 0 & $1 / 16$ & $1 / 8$ \\
$\mathrm{C}$ [V] & 0 & 0 & 30 \\
$\mathrm{E}$ [Aeq] & 1 & 2 & 3 \\
$\mathrm{BP}[\mathrm{Pa}]$ & - & 0 & $6.7 \times 10^{-3}$ \\
\hline
\end{tabular}

eters related to physical phenomena, which will be called "physical parameters" hereafter. Errors in numerical calculations come from approximations to solve partial differential equations and physical recovery models, such as macroparticle number and mass ratio. Uncertainties in physical parameters come from lack of experiments to measure certain parameters, such as thermal accommodation coefficient and lack of knowledge to model complex phenomena like Bohm diffusion. The scope of this paper is the sensitivity analysis of physical parameters, even though consideration of errors will be discussed in the following sub-sections. Table 2 summarizes the physical parameters investigated in this study and their presumed nominal, maximum, and minimum values. This set of parameters essentially comprises all of the physical parameters of our fully kinetic model. The rest of this section serves to explain the detail of the investigated parameters and how the parameter ranges was decided.

\subsubsection{Range of physical parameters related to neutral flow}

In the fully kinetic code, mainly two kinds of parameters have a major impact on neutral propellant flow. The first one is the thermal accommodation coefficient (TAC), which defines the percentage of heat transfer between heavy particles (ions and neutrals) and solid wall surfaces. A TAC of 1.0 means the wall-incident heavy particles will be fully accommodated; that is to say they will be bounced back with a kinetic energy corresponding to the wall temperature, whereas a TAC of 0.0 means the incident particles will be reflected at the wall surface with their initial energy before collision. Since typically heavy particles hit the wall with energy much higher than the wall temperature, the higher TAC slows down the neutral propellant increasing the resi- 
dent time in the channel, which can cause a significant impact on discharge. The experimental work of Kim et al. ${ }^{21)}$ suggests a xenon accommodation coefficient on boron nitride between 0.6 and 0.8 for ion energies between 100 and $400 \mathrm{eV}$ under a controlled experimental configuration. In the real configuration inside a Hall thruster channel, the uncertainty of wall-surface roughness, impurity, temperature, etc. are considered to further broaden the confidence range of the exact TAC value. Therefore, a TAC value of 1.0 was selected as the maximum case, whereas 0.5 was assumed to be the minimum case. Note that, for simplicity, the TAC was assumed to be the same for both ions and neutrals against either the channel wall or anode. Besides, $100 \%$ momentum accommodation assumed that all of the particles leave the wall with the velocity sampled from the Maxwellian distribution. As for the injection condition of the neutrals, their velocity components were randomly assigned according to a half-range Maxwellian distribution.

The second parameter is wall temperature, which essentially affects the energy of the particles accommodating the walls and particles injected from the anode. Direct measurement of the wall surface temperature during the thruster operation is a complicated task, although according to a previous study, ${ }^{22)} 500 \mathrm{~K}$ was assumed to be the maximum (in the sense of neutral resident time) and $900 \mathrm{~K}$ to be the minimum.

\subsubsection{Range of Bohm diffusion coefficient}

The perpendicular component of the classical diffusion coefficient scales with $1 / B^{2}$, and is given as:

$$
D_{\perp}=\frac{1}{1+\omega_{c}^{2} / v^{2}} \frac{k_{B} T_{e}}{m v}
$$

where $\omega_{c}$ is the electron plasma frequency, $v$ is collision frequency, $k_{B}$ is the Boltzmann constant, $T_{e}$ is the temperature, and $m$ is the electron mass. In $\mathrm{E} \times \mathrm{B}$ devices like Hall thrusters, the electron cross-field transport is known to be much greater than what is predicted by the classic diffusion theory. This anomalously enhanced electron transport was first observed by David J. Bohm in 1949, who suggested an empirical coefficient, known as the Bohm coefficient, to account for this anomalous transport. ${ }^{23)}$ Although almost seven decades have passed since its first observation, there does not yet exist a conclusive physical model to be used in numerical simulations. Hence, it is a common practice in Hall thruster simulation that the coefficient of $1 / 16$ suggested by Bohm is used as:

$$
D_{B}=\frac{1}{16} \frac{k_{B} T_{e}}{e B}
$$

where $D_{B}$ is the diffusion coefficient, $B$ is the magnetic field strength, and $e$ is the unit charge. However, it should be noted that this empirical coefficient of $1 / 16$ is not necessarily applicable for different thruster configurations and operation conditions, thus a sensitivity analysis was conducted. The coefficient of $1 / 8$ was assumed to be the maximum, and naturally, elimination of the Bohm diffusion model (e.g., coefficient of zero) was selected to be the minimum.

\subsubsection{Range of cathode coupling voltage}

The voltage utilization efficiency is defined as the ratio of beam voltage to discharge voltage, $\eta_{V}=V_{b} / V_{d}$. As for the beam voltage, it is defined as $V_{b}=V_{d}-V_{c}$, where $V_{c}$ is the cathode coupling voltage. Large cathode coupling voltage is undesirable since it decreases the available potential energy that ions convert into kinetic energy, and thus generate thrust. Cathode coupling voltage in a Hall thruster is usually around $10-30 \mathrm{~V}$. To quantify its effect, a maximum $V_{c}$ of $30 \mathrm{~V}$ was assumed according to the measurement results of thrusters operating under similar conditions, ${ }^{17)}$ and the anode potential applied was simply reduced by $V_{c}$.

\subsubsection{Range of electron injection current}

In the fully kinetic code, electrons are injected through plume boundary cells (see Fig. 1(b)) to sustain the discharge. Note that this electron injection current was not equal to the cathode electron emission current because the cathode was located outside the computational domain. Therefore, the beam neutralization process took place outside the computational domain. Thus, the net electron injection current plus the beam current must equal the discharge current. Since it is nontrivial to know the net electron injection current beforehand, we injected a presumed number of electrons and let the excessive electrons eject from the domain simultaneously. The nominal value of the electron injection current was $2 \mathrm{~A}$, which is equal to double the equivalent xenon propellant current. To quantify its effect, $1 \mathrm{~A}$ and $3 \mathrm{~A}$ were selected as the lower and upper bounds.

\subsubsection{Range of background pressure}

Many studies in literature ${ }^{18,19)}$ conclude that the vacuum chamber background pressure has a remarkable influence on Hall thruster plasma properties and performance parameters, especially for high power applications. ${ }^{24)}$ Thus, it is necessary to correct the experimental results to predict a thruster's in-space performance. The background pressure effect may be considered an experimental measurement error since it increases thrust and efficiency. During the experiments using the UT-62 thruster, the background pressure was kept lower than $3.7 \times 10^{-3} \mathrm{~Pa}$ against a $1.36 \mathrm{mg} / \mathrm{s}$ $\left(+0.27 \mathrm{mg} / \mathrm{s}\right.$ cathode) xenon mass flow rate. ${ }^{12)}$ This pressure was gauged at a point far away from the thruster exit, so it may be assumed that the pressure near the thruster exit plume region is much higher. Therefore, the background pressure upper bound was chosen as $6.7 \times 10^{-3} \mathrm{~Pa}\left(5 \times 10^{-5}\right.$ Torr $)$.

\section{Numerical Results and Discussion}

\subsection{Verification of statistical error 3.1.1. Macroparticle number}

PIC simulations are subject to statistical or "discrete particle" noise. This non-physical effect is related to collisions, and it gives rise to noise-induced particle diffusion. ${ }^{3,25)}$ This numerical heating of the plasma due to statistical noise is proportional to the number of macroparticles per cell, which is proportional to the grid space. Therefore, attention must be paid to convergence with macroparticle size prior to any sensitivity analysis of the physical parameters. Accordingly, the 
macroparticle size convergence of the simulation was studied. The thruster simulated was the UT-62, and the simulation conditions were the same as listed in Table 1 and the "Nominal" physical parameters of Table 2. It is to be noted that the ideal condition is for the macroparticle size to equal to one, which means one simulation particle represents one real particle.

Figure 3 presents the inverse average electron macroparticle number against the simulated discharge current. It should be noted that the simulated discharge current has a linear relationship with the number of particles per grid. The parallel decrease in discharge current and macroparticle size is because of the noise-induced particle diffusion. This result suggests that the simulation result converges almost linearly; therefore, the size of $1 \times 10^{7}$ was selected as a good trade-off between the numerical error and the computational cost for this specific thruster. Note that the discharge current extrapolated to macroparticle size equals one does not match the measurement result perfectly, which is natural because various measurement and model uncertainties in physical parameters are yet to be accounted for.

\subsubsection{Grid spacing}

The effect of grid spacing on the discharge plasma and wall sheath structure was investigated by Cho et al. ${ }^{12)}$ It was concluded that a grid spacing of $0.2 \mathrm{~mm}$ is sufficient to accurately capture the sheath potential drop. Some additional simulations were conducted to examine the effect of grid size on the simulation results, which is shown by Table 3. Furthermore, the effect of thermal accommodation coefficient, anode and wall temperatures (Case A) was also simulated on a smaller grid spacing of $0.1 \mathrm{~mm}$ along with the nominal grid spacing $(0.2 \mathrm{~mm})$. It can be seen in Table 3 that, for both of the grid spacings, discharge current, thrust, and wall erosion rate are considerably affected by the change in thermal accommodation coefficient, and anode/wall temperature. This result means that the effect observed in thruster per-

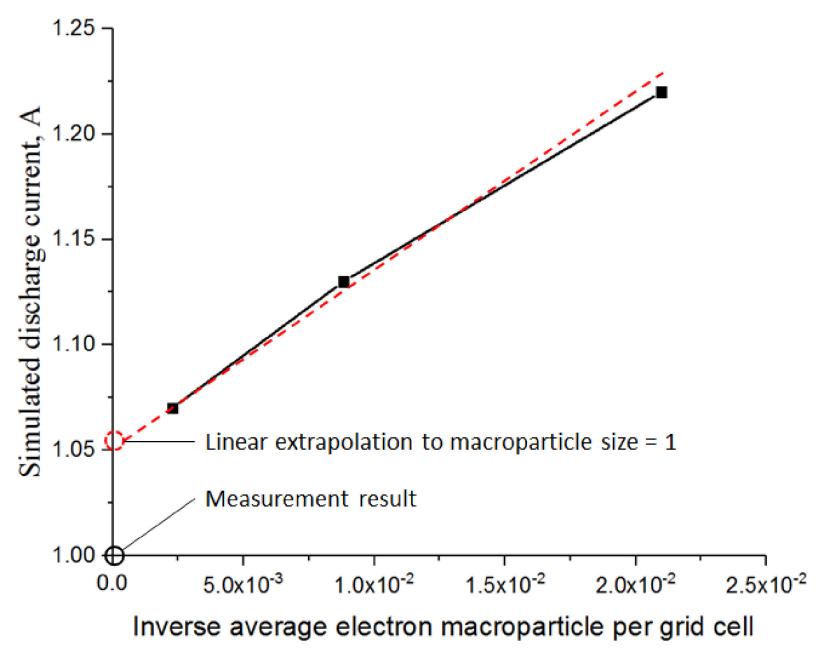

Fig. 3. Convergence of discharge current against electron macroparticle size.

The linear fit shows the result extrapolated to the ideal condition of size $=1$. formance is not caused simply by the choice of grid size. Even though the grid spacing selected is larger than the Debye length causing uncertainty to the sheath modeling, it seems to have a minor impact on the sensitivity analysis.

\subsection{Thruster performance}

Figure 4 summarizes the results of the one-factor-at-atime sensitivity analysis for thrust and discharge current and compares them to experimental results. It should be noted that a water-cooling system was used for ground testing of the UT-62; however, the experimental results were not evaluated under thermal equilibrium conditions, but measured under cold-start conditions. It was observed that the sensitivity of A, neutral flow (thermal accommodation coefficient and anode/wall temperatures), and B, Bohm diffusion coefficient, were by far the most influential. It was found that the uncertainties in physical parameters arising from the neutral flow (thermal accommodating coefficient and anode/ wall temperatures) were just as influential as the Bohm diffusion coefficient. This was one of the most important findings of this study because less attention has been paid to them so far. This suggests that improving (narrowing) the confidence

Table 3. Effect of thermal accommodation coefficient, anode and wall temperatures on smaller grid spacing.

\begin{tabular}{clccc}
\hline Grid spacing & Case A & $F[\mathrm{mN}]$ & $I_{d}[\mathrm{~A}]$ & $\begin{array}{c}\text { Max erosion rate } \\
{[\mathrm{mm} / \mathrm{kh}]}\end{array}$ \\
\hline \multirow{2}{*}{$0.1 \mathrm{~mm}$} & Nom & 18.4 & 1.2 & 21.5 \\
& Min & 17.2 & 1.1 & 19.1 \\
\multirow{3}{*}{$0.2 \mathrm{~mm}$} & Max & 19.3 & 1.3 & 24.7 \\
& Nom & 15.7 & 1.1 & 20.7 \\
& Min & 14.6 & 1.0 & 16.7 \\
& Max & 17.0 & 1.2 & 22.7 \\
\hline
\end{tabular}

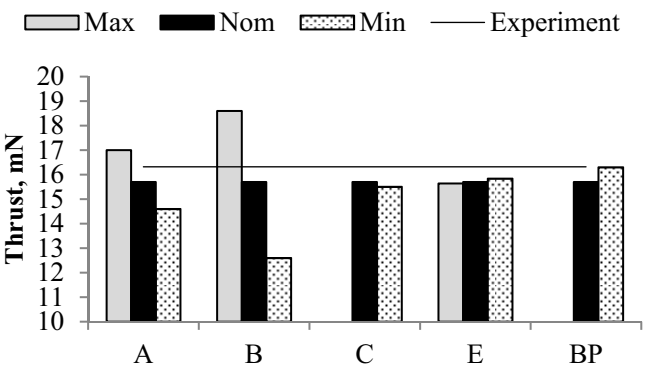

(a) Thrust

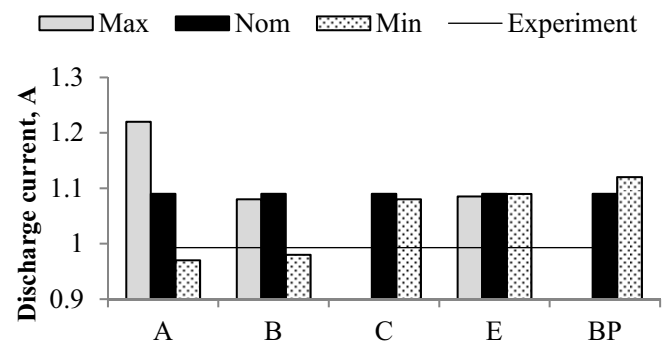

(b) Discharge current

Fig. 4. Summary of the one-at-a-time sensitivity analysis results for thrust and discharge current.

Note the "nom" nominal condition is displaying the same result for all cases. 
Table 4. Simulation results for A, B, and BP.

\begin{tabular}{|c|c|c|c|c|c|c|c|}
\hline & \multirow{2}{*}{ Case } & \multirow{2}{*}{ Nom } & \multicolumn{2}{|c|}{ A } & \multicolumn{2}{|c|}{ B } & \multirow{2}{*}{$\begin{array}{c}\text { BP } \\
\text { Max }\end{array}$} \\
\hline & & & Min & Max & Min & Max & \\
\hline \multirow{6}{*}{$\begin{array}{l}\text { Sensitivity } \\
\text { (input) } \\
\text { parameters }\end{array}$} & $T_{\text {co }}$ & 0.9 & 0.5 & 1.0 & 0.9 & 0.9 & 0.9 \\
\hline & $E_{\text {co }}$ & 0.9 & 0.5 & 1.0 & 0.9 & 0.9 & 0.9 \\
\hline & $T_{\text {anode }}[\mathrm{K}]$ & 500 & 900 & 500 & 500 & 500 & 500 \\
\hline & $T_{\text {wall }}[\mathrm{K}]$ & 700 & 900 & 500 & 700 & 700 & 700 \\
\hline & Bohm & $1 / 16$ & $1 / 16$ & $1 / 16$ & 0 & $1 / 8$ & $1 / 16$ \\
\hline & $\mathrm{Bp}[\mathrm{Pa}]$ & 0 & 0 & 0 & 0 & 0 & $6.7 \times 10^{-3}$ \\
\hline \multirow{10}{*}{$\begin{array}{l}\text { Output } \\
\text { parameters } \\
\text { (numerical } \\
\text { simulation } \\
\text { results) }\end{array}$} & $F[\mathrm{mN}]$ & 15.7 & 14.6 & 17.0 & 18.6 & 12.6 & 16.3 \\
\hline & $I_{d}[\mathrm{~A}]$ & 1.09 & 0.97 & 1.22 & 0.98 & 1.08 & 1.12 \\
\hline & $I_{b 1}[\mathrm{~A}]$ & 0.78 & 0.69 & 0.87 & 0.78 & 0.74 & 0.80 \\
\hline & $I_{b 2}[\mathrm{~A}]$ & 0.11 & 0.10 & 0.12 & 0.10 & 0.10 & 0.11 \\
\hline & $I_{n}[\mathrm{~A}]$ & 0.17 & 0.26 & 0.07 & 0.16 & 0.21 & 0.22 \\
\hline & $\Delta$ & 0.03 & 0.04 & 0.02 & 0.04 & 0.07 & 0.02 \\
\hline & $\eta_{t}[\%]$ & 27.9 & 27.0 & 29.0 & 43.5 & 17.9 & 29.2 \\
\hline & $\eta_{E}[\%]$ & 60 & 56 & 61 & 64 & 60 & 59 \\
\hline & $\eta_{u}[\%]$ & 83 & 74 & 93 & 84 & 79 & 85 \\
\hline & $\eta_{a}[\%]$ & 56 & 65 & 51 & 81 & 38 & 58 \\
\hline
\end{tabular}

range of uncertainties in physical parameters related to neutral flow will be as beneficial as that of the Bohm diffusion model for the accuracy of fully kinetic simulation.

The rest of this section presents the details of the top three influential physical parameters: parameters related to neutral flow, Bohm diffusion coefficient, and background pressure.

\subsubsection{Effect of thermal accommodation coefficient, anode and wall temperatures}

Table 4 shows the detailed results of the sensitivity analysis for A, B, and BP physical parameters. The symbol "nom" represents the nominal case, while $\mathrm{A}_{\max }, \mathrm{A}_{\min }$ are respectively the maximum and minimum cases. It can be seen from Table 4 that any change in thermal accommodation coefficient and temperature mostly affects the ionization process, represented by propellant efficiency $\Phi_{P}$. This was caused by the increase in propellant residence time in the discharge channel due to the slower flow speed of unionized propellant, the origination of which is increase in thermal accommodation coefficient and lower wall temperature. The discharge current and ion beam current increase monotonically from the minimum case to the maximum case; whereas the unionized neutral propellant current $I_{n}$ decreases. The plasma properties illustrated by Fig. 5 support this understanding in that the plasma density of the maximum case $\left(A_{\max }\right)$ was much higher than that of the minimum case $\left(A_{\min }\right)$.

Notice that the ion density distribution is not uniform in the radial direction. We believe that the concentration of ion density in the vicinity of outer channel was caused by the shape of the magnetic field lines, which generated equipotential lines slanted towards the outer channel. This leads to a potentially larger drop in the sheath near the outer wall as compared to the inner wall. As a result, ions are accelerated from the bulk plasma toward the outer wall where they become neutral after recombination, and increase neutral density, electron-neutral collision frequency, and thus ion/electron density near the outer wall. This may also explain why the neutral temperature difference between $\mathrm{A}_{\max }$ and $\mathrm{A}_{\min }$ is two orders of magnitude large. It is worth mentioning that
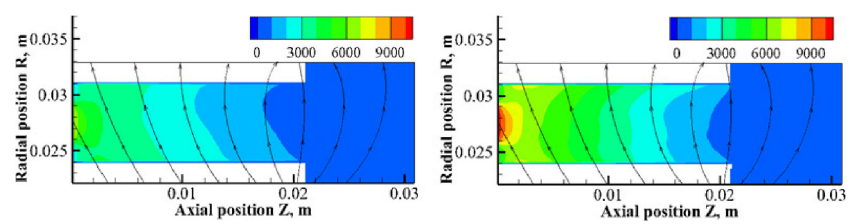

(a) Neutral density distribution $\left(10^{16} \mathrm{~m}^{-3}\right)$
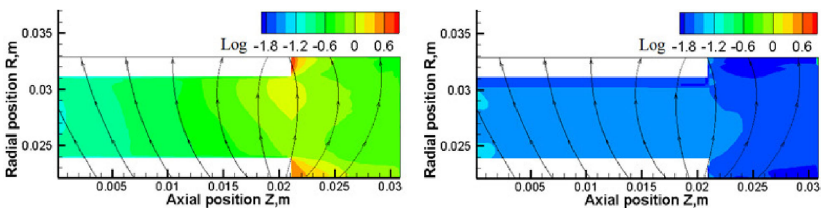

(b) Neutral temperature $(\mathrm{eV})$
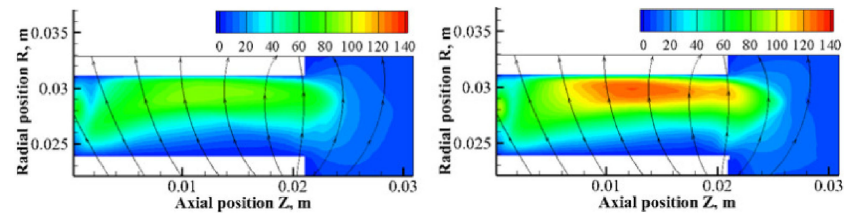

(c) Plasma ion density distribution $\left(10^{16} \mathrm{~m}^{-3}\right)$
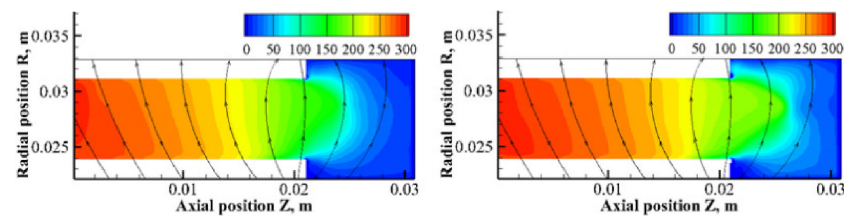

(d) Potential distribution (V)
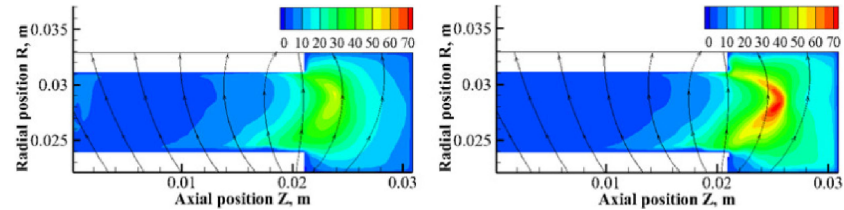

(e) Electron temperature $(\mathrm{eV})$

Fig. 5. Simulated plasma properties for the accommodation coefficient $\&$ anode/wall temperature $\left(\mathrm{A}_{\max }\right.$ and $\left.\mathrm{A}_{\min }\right)$.

Plots on the left belong to $A_{\text {min }}$ case. Plots on the right belong to $A_{\max }$ case. Lines with arrows represent the applied magnetic field.

not only the propellant utilization, but also the beam efficiency $\left(\eta_{a}\right.$ in Table 4$)$ and the shape of the equipotential lines (Fig. 5(d)) changed slightly. This was considered to be caused due to the change in electron cross-field transport; namely, the higher electron temperature and density of the maximum case leading to an increase in electron diffusion and diffusive plasma distribution. In addition, it should be noted that the electron temperature is controlled by the electron-neutral collisions since these collisions are the primary contributor to electron energy loss. The enhanced electron temperature in the maximum case $\left(\mathrm{A}_{\max }\right)$ is because the neutral density, and thus electron energy loss due collisions, outside the channel was reduced in contrast to that inside the channel.

\subsubsection{Effect of Bohm diffusion coefficient}

The differences in plasma properties are presented in Fig. 6. This time, the beam efficiency $\eta_{a}$ was the efficiency most influenced. From Table 4, it was found that thruster performance deteriorated as the Bohm coefficient increased, and thus, cross-field electron transport increased due to the 

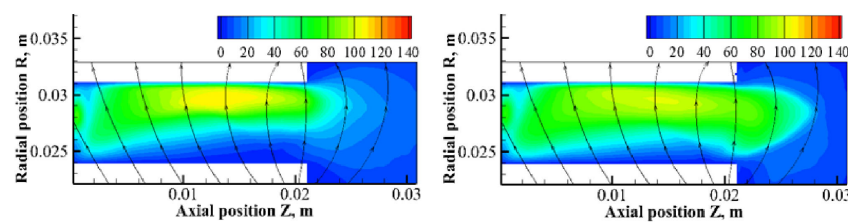

(a) Plasma ion density distribution $\left(10^{16} \mathrm{~m}^{-3}\right)$
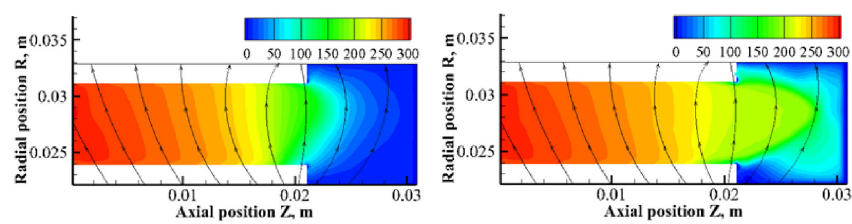

(b) Potential distribution (V)
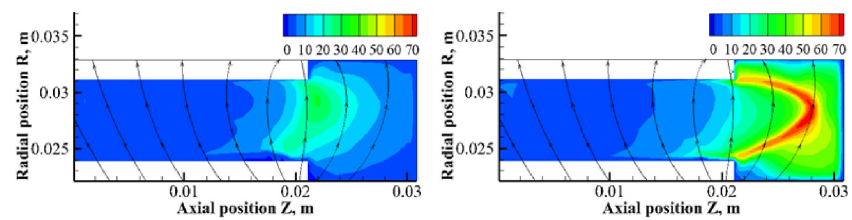

(c) Electron temperature $(\mathrm{eV})$

Fig. 6. Simulated plasma properties for the Bohm diffusion coefficient $\left(\mathrm{B}_{\max }\right.$ and $\left.\mathrm{B}_{\min }\right)$

Plots on the left belong to $\mathrm{B}_{\min }$ case. Plots on the right belong to $\mathrm{B}_{\max }$ case.

increasingly diverse beam. The electron current (discharge current minus total beam current) also monotonically increased from the minimum case $\left(\mathrm{B}_{\min }\right)$ to the maximum case $\left(\mathrm{B}_{\max }\right)$, which was apparently caused by the increased electron transport.

Unlike the A parameter cases, the major differences were found to be the shape of the equipotential lines and electron temperature. Obviously, these changes were caused by the change in electron cross-field transport due to the artificially increased/decreased diffusion due to the Bohm diffusion model.

\subsubsection{Background pressure effect}

To set various background pressures inside the channel, the background pressure was controlled by a neutral xenon flow rate from the plume boundary cells as shown in Fig. 1. The background pressure at the plume boundary was assumed to be spatially uniform, and the amount of the background neutral injection was determined to be equal to the difference between the presumed background pressure and the pressure of the plume boundary cells. Thus, no background neutrals were injected into a cell when the pressure in that cell was higher than the presumed background pressure. The background neutrals were assumed to be thermalized to the vacuum chamber wall temperature of $300 \mathrm{~K}$.

Figure 7 shows the simulation results of thrust and anode efficiency under different background pressure conditions. The existence of background pressure artificially increased the thrust and anode efficiency as reported in a previous study. ${ }^{24)}$ It was also observed that the simulated thrust and efficiency come close to the results measured, when the background pressure increases, which might explain the discrepancies between the simulation and experiment.

The breakdown of the efficiency in Table 4 shows that the

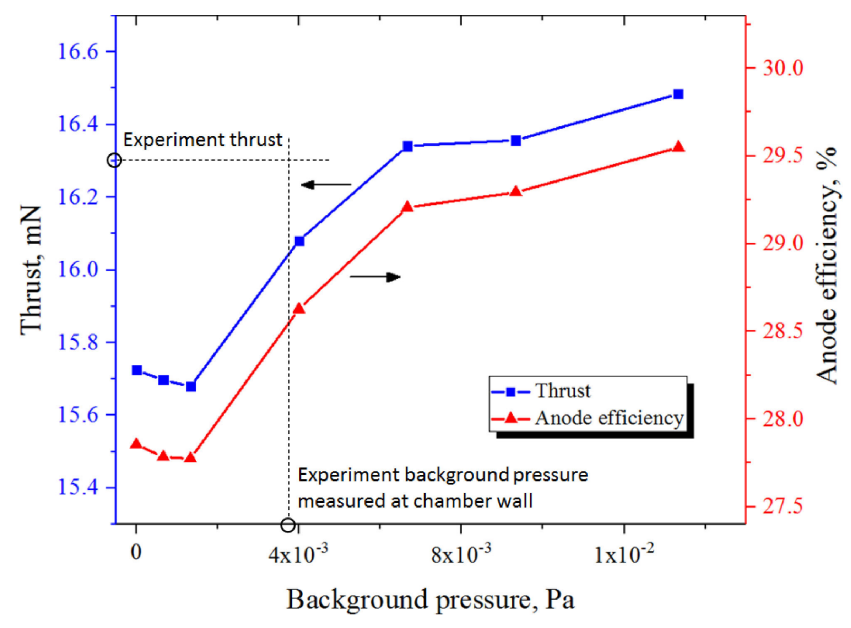

Fig. 7. Simulated thrust and anode efficiency under different background pressure conditions.

$\square \operatorname{Max}$ Nom $\mathrm{QMin}$

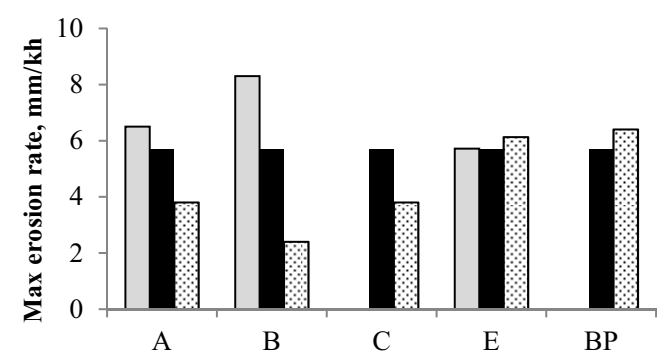

(a) Inner wall

$\square$ Max $\square$ Nom $\mathrm{OMin}$

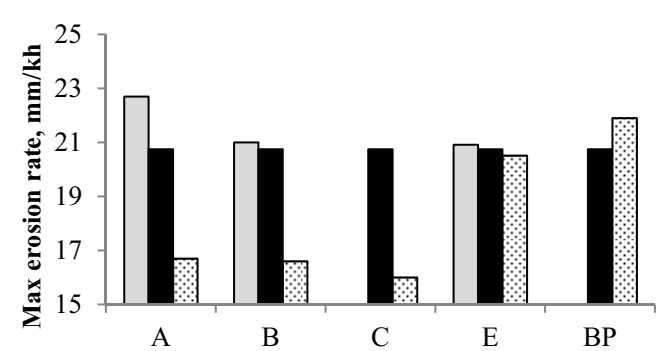

(b) Outer wall

Fig. 8. Summary of the one-at-a-time sensitivity analysis results for the maximum channel-wall erosion rate.

background pressure artificially increases the thrust efficiency by increasing propellant efficiency as a result of injecting background neutrals. It also shows that the background pressure not only increases the thrust, but also the discharge current, which means it has a relatively small impact on the thrust-to-power ratio compared to the specific impulse.

It is to be noted that there was no change in the thruster performance for background pressures presumed lower than $1.33 \times 10^{-3} \mathrm{~Pa}$, which means the pressure of the plume boundary cells cannot be lower than $1.33 \times 10^{-3} \mathrm{~Pa}$ due to the unionized neutrals escaping from the thruster.

\subsection{Channel wall erosion}

Lifetime is another critically important performance consideration for Hall thrusters. Usually, the lifetime of a Hall 


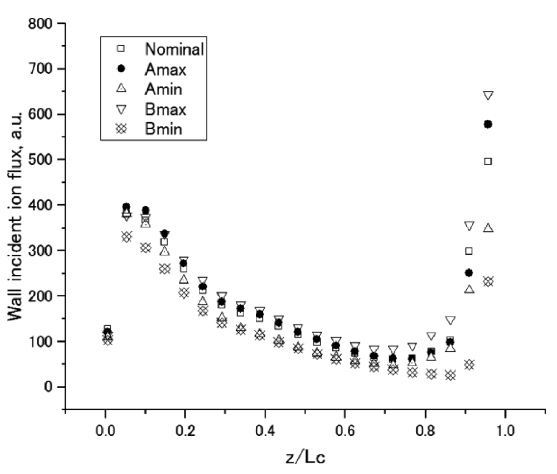

(a) Inner-wall incident ion flux

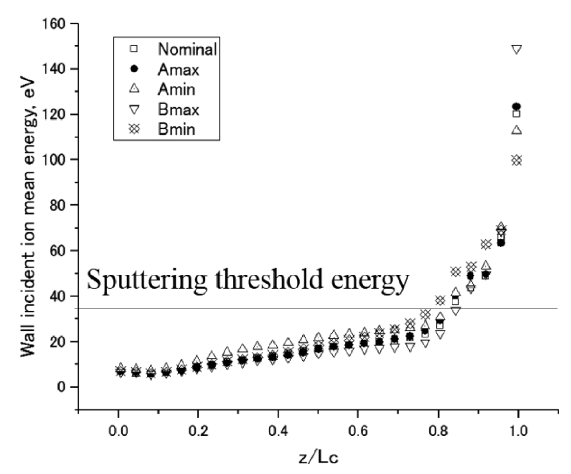

(c) Inner-wall incident ion mean energy

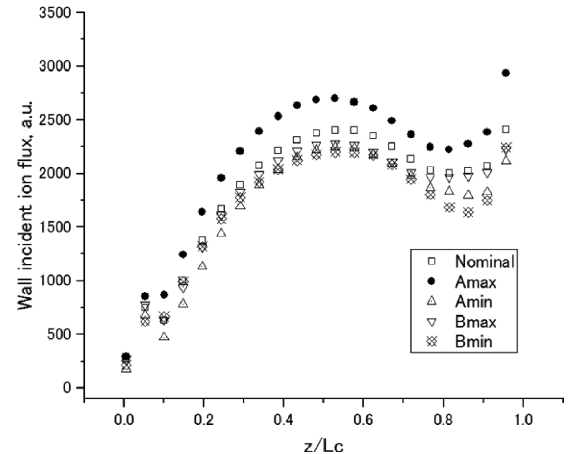

(b) Outer-wall incident ion flux

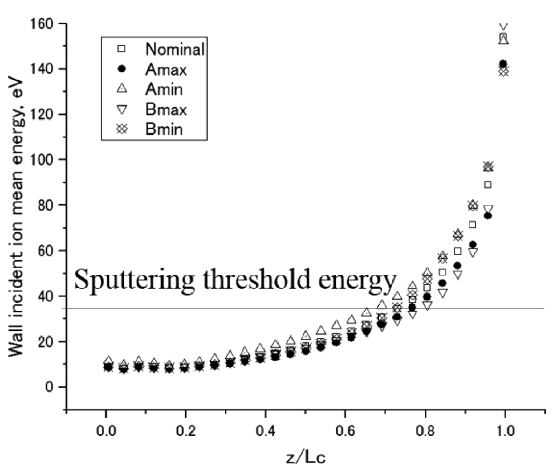

(d) Outer-wall incident ion mean energy

Fig. 9. Simulated wall-incident ion properties distribution.

thruster is determined by the erosion rate of the discharge channel walls, which is caused by sputtering due to the high-energy ion bombardment. Figure 8 summarizes the simulated wall erosion rate results calculated according to the wall-incident ion flux and energy. The channel wall material (boron nitride, $\mathrm{BN}$ ) threshold energy of ion sputtering was assumed to be 35 electron volts, and the Yamamura model was used to approximate the sputtering yield. ${ }^{26)}$ Since the wall erosion rates were not uniformly distributed throughout the channel, only the maximum rate was shown as the bottleneck of the thruster lifetime.

The results indicate that the thruster lifetime is more sensitive to the physical parameters than the discharge current or thrust. The influential parameters are the ones related to neutral flow and the Bohm diffusion coefficient; however, the impact of cathode-coupling voltage loss became nontrivial in contrast to its impact on the thrust efficiency. To elucidate the cause of the wall erosion, the wall-incident ion properties of nominal, parameter A, and parameter B cases are shown by Fig. 9. For all of the cases and the inner-channel and outer-channel, the peak of the wall erosion rate placed at the channel exit (not shown) having the highest ion flux and energy. On the contrary, the upstream channel was essentially erosion-free because the incident ion energy was lower than the BN sputtering threshold energy. According to the comparison between the five cases listed, it was found that both ion flux and energy contribute to the change in channel wall erosion rate. Although the discrepancy of the ion flux was larger than that of the ion energy, the difference in ion energy was also important because of the non-linearity of the sputtering rate near the threshold.

\section{Conclusion}

A sensitivity analysis was performed to evaluate the importance of the uncertainties associated with physical parameters for better predicting the accuracy of Hall thruster simulation. Fully kinetic code physical parameters (thermal accommodation coefficient, anode/wall temperatures, Bohm diffusion coefficient, electron injection current, cathode coupling voltage, and background pressure) were investigated. The impact of each physical parameter on thrust efficiency and wall erosion rate was quantified one-by-one over a presumed possible range. The simulation was conducted for a laboratory model magnetic-layer Hall thruster.

The uncertainties in the physical parameters reasonably account for the discrepancies between the measured and simulated results. Furthermore, the results indicate that the model parameters have a more severe impact on wall erosion than thrust efficiency. Among the sources of uncertainties, the Bohm diffusion coefficient and uncertainties related to the neutral flow (thermal accommodation coefficient and anode/wall temperatures) were by far the most influential for both thrust efficiency and wall erosion; whereas the impact of other uncertainties including electron injection current, cathode coupling voltage, and background pressure was minor. It was found that uncertainties in physical parameters related to the neutral flow has a comparable influence to that of Bohm diffusion, which suggests that accurately identifying not only the Bohm diffusion coefficient, but also the thermal accommodation coefficient and anode/wall temperatures is essential for better predictive modeling of Hall thrusters. 


\section{References}

1) Zhurin, V. V., Kaufman, H. R., and Robinson, R. S.: Physics of Closed Drift Thrusters, Plasma Sources Sci. Technol., 8, 1 (1999), pp. R1R20.

2) Komurasaki, K. and Arakawa, Y.: Two-dimensional Numerical Model of Plasma Flow in a Hall Thruster, J. Propul. Power, 11, 6 (1995), pp. 1317-1323

3) Szabo, J. J.: Fully Kinetic Numerical Modeling of a Plasma Thruster, PhD Thesis, Massachusetts Institute of Technology, Cambridge, 2001.

4) Fife, J. M.: Hybrid-PIC Modeling and Electrostatic Probe Survey of Hall Thrusters, $\mathrm{PhD}$ Thesis, Massachusetts Institute of Technology, Cambridge, 1998.

5) Mikellides, I. G., Katz, I., Hofer, R. R., and Goebel, D. M.: Magnetic Shielding of Walls from the Unmagnetized Ion Beam in a Hall Thruster, Appl. Phys. Lett., 102, 2 (2013), id. 023509, 5 pp.

6) Adam, J. C., Heron, A., and Laval, G.: Study of Stationary Plasma Thrusters Using Two-dimensional Fully Kinetic Simulations, Phys. Plasmas, 11, 1 (2004), pp. 295-305.

7) Fife, J., Martinez-Sanchez, M., and Szabo, J.: A Numerical Study of Low-frequency Discharge Oscillations in Hall Thrusters, Proceedings of 33rd AIAA Joint Propulsion Conference, No. 97-3051, 1997.

8) Parra, F. I., Ahedo, E., Fife, J. M., and Martinez-Sanchez, M.: A Twodimensional Hybrid Model of the Hall Thruster Discharge, J. Appl. Phys., 100, 023304 (2006).

9) Watanabe, H., Nakano, M., Kajimura, Y., and Funaki, I.: Feasibility Study on Numerical Life Qualification of Ion Thruster's Ion Optics Using the JIEDI Tool, Trans. JSASS Aerospace Technology Japan, 12, ists29 (2014), pp. Pb_65-Pb_72.

10) Yamamoto, N., Nakagawa, T., Komurasaki, K., and Arakawa, Y.: Discharge Plasma Fluctuations in Hall Thrusters, Vacuum, 65, 3-4 (2002), pp. 375-381.

11) Yokota, S., Komurasaki, K., and Arakawa, Y.: Plasma Density Fluctuation Inside a Hollow Anode in an Anode-layer Hall Thruster, Proceedings of 42nd AIAA/ASME/SAE/ASEE Joint Propulsion Conference \& Exhibit, AIAA-2006-5170.

12) Cho, S., Komurasaki, K., and Arakawa, Y.: Kinetic Particle Simulation of Discharge and Wall Erosion of a Hall Thruster, Phys. Plasmas, 20, 6 (2013), 063501, 12 pp.

13) Cho, S., Watanabe, H., Kubota, K., Iihara, S., Honda, K., Fuchigami, K., Uematsu, K., and Funaki, I.: Parametric Kinetic Simulation of an IHI High Specific Impulse SPT-Type Hall Thruster, Proceedings of 50th AIAA/ASME/SAE/ASEE Joint Propulsion Conference \& Exhibit, AIAA-2014-3426.

14) Brown, D. L.: Investigation of Low Discharge Voltage Hall Thruster Characteristics and Evaluation of Loss Mechanisms, PhD Thesis, University of Michigan, Ann Arbor, 2009.

15) Hofer, R. R., Katz, I., Mikellides, I. G., and Gamero-Castano, M.: Heavy Particle Velocity and Electron Mobility Modeling in HybridPIC Hall Thruster Simulations, AIAA-2006-4658.

16) Hofer, R. R., Katz, I., Mikellides, I. G., Goebel, D. M., Jameson, K. K., Sullivan, R. M., and Johnson, L. K.: Efficacy of Electron Mobility Models in Hybrid-PIC Hall Thruster Simulations, AIAA-2008-4924.

17) Jameson, K. K., Goebel, D. M., Hofer, R. R., and Watkins, R. M.: Cathode Coupling in Hall Thrusters, Proceedings of 30th International Electric Propulsion Conference, IEPC-2007-278.

18) Randolph, T., Kim, V., Kaufman, H., Kozubsky, K., Zhurin, V., and Day, M.: Facility Effects on Stationary Plasma Thruster Testing, Proceedings of 23rd International Electric Propulsion Conference, IEPC1993-093.

19) Kamhawi, H., Huang, W., Haag, T., and Spektor, R.: Investigation of the Effects of Facility Background Pressure on the Performance and Voltage-Current Characteristics of the High Voltage Hall Accelerator, Proceedings of 33rd International Electric Propulsion Conference, IEPC-2013-446.

20) Hora, S. C.: Aleatory and Epistemic Uncertainty in Probability Elicitation with an Example from Hazardous Waste Management, Reliability Eng. Syst. Safety, 54 (1996), pp. 217-223.

21) Kim, V., Semenov, A., and Shkarban, I.: Investigation of the Accelerated Ions Energy Accommodation under Their Impingement with Solid Surfaces, Proceedings of 38th AIAA/ASME/SAE/ASEE Joint Pro- pulsion Conference \& Exhibit, AIAA-2002-4110.

22) Karadag, B., Cho, S., and Funaki, I.: Numerical Investigation of Thermal Accommodation Coefficient and Temperature Sensitivity in a Hall Thruster, Proceedings of Plasma Conference 2014, 19pD2-5.

23) Bohm, D.: The Characteristics of Electrical Discharges in Magnetic Fields, Guthrie, A. and Wakerling, R. K. (ed.), McGraw-Hill, New York, 1949, pp.77-86.

24) Byers, D. and Dankanich, J. W.: A Review of Facility Effects on Hall Effect Thrusters, Proceedings of 31st International Electric Propulsion Conference, IEPC-2009-076.

25) Hockney, R. W. and Eastwood, J. W.: Computer Simulation Using Particles, Institute of Physics Publishing, Bristol and Philadelphia, 1988.

26) Yamamura, Y. and Tawara, H.: Energy Dependence of Ion-Induced Sputtering Yields from Monatomic Solids at Normal Incidence, Atomic Data and Nuclear Data Tables, 62, 2 (1996), pp. 149-253.

\section{Appendix}

\section{A.1. Efficiency analysis}

The efficiency breakdown scheme proposed by Brown ${ }^{14)}$ was used to analyze thrust generation processes influenced by the physical parameters. ${ }^{9)}$ First of all, anode efficiency $\eta_{t}$, the indicator of overall energy conversion of the thrusterhead, can be divided into energy efficiency $\eta_{E}$, propellant efficiency $\eta_{u}$, and beam efficiency $\eta_{a}$ as:

$$
\eta_{t}=\frac{F^{2}}{2 \dot{m} P_{d}}=\eta_{E} \eta_{u} \eta_{a}
$$

where, $F$ is the thrust, $\dot{m}$ is anode mass flow rate, and $P_{d}$ is discharge power.

Energy efficiency $\eta_{E}$ represents the ratio of energy deposited on the propellant $P_{j e t}$ to the discharge power as:

$$
\eta_{E}=\frac{P_{j e t}}{P_{d}}=\frac{\frac{1}{2} \dot{m}\left\langle\overline{v^{2}}\right\rangle_{m}}{V_{d} I_{d}}
$$

where, $\left\langle\overline{v^{2}}\right\rangle_{m}$ is the mass weighted average of the square of the propellant velocity, $V_{d}$ is discharge voltage, and $I_{d}$ is discharge current.

Propellant efficiency $\eta_{u}$ represents the relationship between the propellant momentum and kinetic energy. It is used mainly to show the thrust loss due to the unionized propellant ejected:

$$
\eta_{u}=\frac{\langle\bar{v}\rangle_{m}^{2}}{\left\langle\overline{v^{2}}\right\rangle_{m}}
$$

where, $\langle\bar{v}\rangle_{m}$ is the mass weighted average velocity.

Finally, beam efficiency $\Psi_{B}$ shows the thrust vector loss due to beam divergence:

$$
\eta_{a}=\left(\frac{F}{\dot{m}\langle\bar{v}\rangle_{m}}\right)^{2}
$$

All of the efficiencies were computed straight-forwardly probing the simulation particles and their variation caused by the physical parameters examined.

\section{A.2. Discharge current oscillation amplitude}

Discharge current oscillation amplitude $(\Delta)$ is defined as: 
Trans. Japan Soc. Aero. Space Sci., Vol. 60, No. 2, 2017

$$
\Delta=\frac{R M S}{\overline{I_{d}}}=\frac{1}{\overline{I_{d}}} \sqrt{\int_{0}^{\tau} \frac{\left(I_{d}-\overline{I_{d}}\right)^{2}}{\tau}}, \quad\left(\overline{I_{d}}=\frac{\int_{0}^{\tau} I_{d}}{\tau}\right)
$$

where, $R M S$ is the root mean square, $I_{d}$ is discharge current, and $\tau$ is time. An oscillation amplitude of $\Delta<0.2$ is desirable due to power processing unit (PPU) constraints.

Y. Ohkawa

Associate Editor 